\title{
First Results From Sleuth: The Palomar Planet Finder
}

\author{
Francis T. O’Donovan*, David Charbonneau* and Lewis Kotredes* \\ *California Institute of Technology, M/C 105-24, Pasadena, CA 91125
}

\begin{abstract}
We discuss preliminary results from our first search campaign for transiting planets performed using Sleuth, an automated $10 \mathrm{~cm}$ telescope with a 6 degree square field of view. We monitored a field in Hercules for 40 clear nights between UT 2003 May 10 and July 01, and obtained an rms precision (per 15-min average) over the entire data set of better than $1 \%$ on the brightest 2026 stars, and better than $1.5 \%$ on the brightest 3865 stars. We identified no strong candidates in the Hercules field. We conducted a blind test of our ability to recover transiting systems by injecting signals into our data and measuring the recovery rate as a function of transit depth and orbital period. About $85 \%$ of transit signals with a depth of $0.02 \mathrm{mag}$ were recovered. However, only $50 \%$ of transit signals with a depth of $0.01 \mathrm{mag}$ were recovered. We expect that the number of stars for which we can search for transiting planets will increase substantially for our current field in Andromeda, due to the lower Galactic latitude of the field.
\end{abstract}

\section{ACQUISITION AND ANALYSIS OF SLEUTH OBSERVATIONS}

Sleuth ${ }^{1}$, located at Palomar Observatory in Southern California, is the third transit-search telescope in our network which comprises STARE ${ }^{2}$ (PI: T. Brown, located in Tenerife), and PSST (PI: E. Dunham, located in northern Arizona). Sleuth is an f/2.8 lens with a $10 \mathrm{~cm}$ aperture that images a $6^{\circ} \times 6^{\circ}$ field of view onto a $2048 \times 2048$ back-illuminated CCD camera. Sleuth conducts nightly observations with an SDSS r' filter, but also gathers color images in g', i' \& z' during new moon. Sleuth automatically adjusts the focus for changes in temperature and filter. A separate f/6.3 lens feeds the guide camera. The automated observations, including operation of the clamshell enclosure, are controlled by a workstation running Linux. In the event of threatening weather, the on-site night assistant for the 200" telescope can close the system remotely, and an observatory weather station provides additional protection. At dawn, the night's data are automatically compressed and sent by ftp to our workstation at Caltech.

Between UT 2003 May 10 and July 01, we monitored approximately 10,000 stars $(9<R<16)$ in a field in Hercules. Figure 1 shows the calculated rms error in our photometry. We applied the STARE photometry code (written by T. Brown [HAO/NCAR] and with adaptations by G. Mandushev [Lowell Obs.]) to calibrate and perform weighted-aperture photometry upon the images. We subsequently combined the time series for each star for all nights. These light curves were then processed through a

\footnotetext{
1 http: //www.astro.caltech.edu/ ftod/sleuth.html

2 http://www.hao.ucar.edu/public/research/stare/stare.html
} 

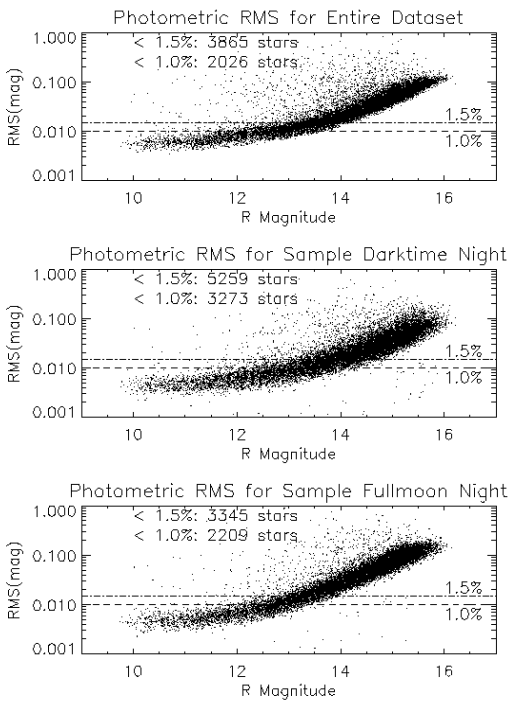

FIGURE 1. The calculated rms as a function of $\mathrm{R}$ magnitude for the 10,000 brightest stars in our Hercules field for three subsets of data: (upper panel) all observations; (center panel) observations from the night of June 29th UT during new moon; (lower panel) observations from the night of June 14th UT during full moon. The numbers of stars with rms below $1.0 \%$ and $1.5 \%$ for each dataset are shown.

cleaning pipeline to remove discrepant data and correlated variations in magnitude. Finally, the data were averaged in 15 minute bins to produce final light curves.

We then used the box-fitting algorithm of Kovács et al. [1] to search these light curves for transits with periods ranging from 1.5 to 7.5 days. This program assigned a Signal Detection Efficiency (SDE - see [1]) to the star, based on the significance of the transit detection. Figure 2 shows a histogram of the SDEs of the thousand brightest stars in the Hercules field, and compares it with the SDEs of a thousand simulated light curves with a Gaussian noise distribution of the same mean and variance as the original timeseries.

We also tested the transit search code by inserting simulated transits into our photometric data and attempting to recover these signals - an example of such an injected transit is shown in Figure 3. The recovery rate of these transits is about $85 \%$ for transits with a depth of $0.02 \mathrm{mag}$, and about $50 \%$ for $0.01 \mathrm{mag}$ transits.

\section{THE NEXT STEPS}

Throughout September and October 2003, we gathered photometric data on a field in Andromeda. The lower Galactic latitude of this field $\left(b=-16^{\circ}\right.$ versus $b=+40^{\circ}$ for the Hercules field) will lead to a significantly larger number of stars that we can search 


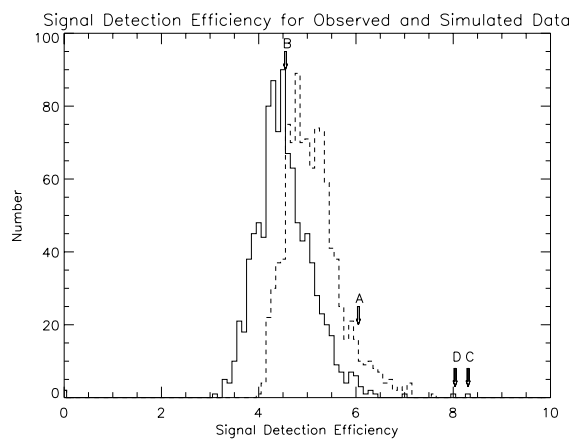

FIGURE 2. The solid line shows the histogram of derived values for the Signal Detection Efficiency (SDE) for the best-fit transit signal for each of the 1000 brightest stars. The histogram of derived SDE values for our simulated data set is plotted as the dashed line. The phased time series for two stars with large SDE values (indicated as C and D on the plot) are shown in Figure 4. We also inserted transits into the data and attempted to recover these: phased light curves of two such recovered systems are shown in Figure 3, and the resulting SDE values are indicated on the plot for the actual data (A) and the simulated data (B).
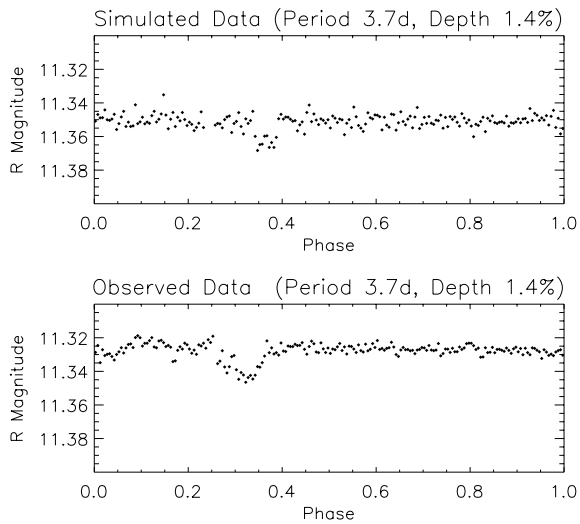

FIGURE 3. As a test of our ability to recover transit signals, we injected transits of varying period and depth into the data, as well as into simulated data. The recovered 1.4\%-deep transits for a 3.7 day period for two such stars are shown for the simulated data (upper panel) and actual data (lower panel). The SDE values for these recoveries are shown in Figure 2.

for transiting planets. The Andromeda data also benefit from a firmware upgrade to our guider, which removed the large night-to-night pixel offsets that the Hercules data were subject to. Whereas the typical offsets in Hercules were 20 pixels, in our current field these offsets rarely exceed 5 pixels, and guiding within a single night is often good to 3 pixels.

We will perfom more detailed tests of our transit detection algorithm to calibrate and 

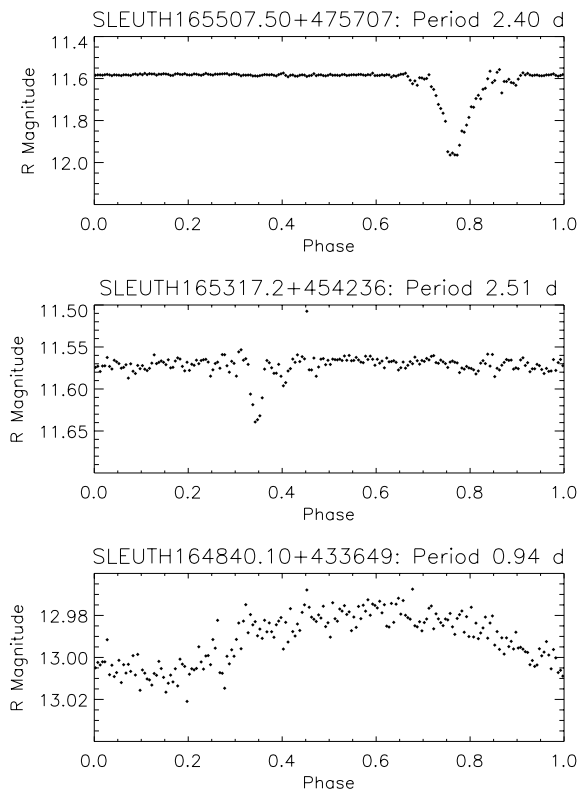

FIGURE 4. The phased light curve for: (C - upper panel) a grazing-incidence eclipsing binary system, and (D - center panel) a low-amplitude eclipsing system. The SDE values for these systems are shown in Figure 2. The phased light curve of a detected low-amplitude variable stars is also shown (lower panel).

improve the detection probability. We are concerned that the cleaning pipeline itself may be introducing photometric correlations between the target stars, and thus reducing our detection capability. We intend to introduce simulated transits into the data before cleaning, and verify that this processing does not remove these events.

Example Sleuth light curves of eclipsing binaries are shown in Figure 4. Figure 4 also shows the light curve of a variable star. We intend to compile a catalog of such binaries and variables. A dominant concern for any transit survey is the rejection of false positives, i.e. systems containing an eclipsing binary that mimic the photometric light curve of a transiting planet. (The eclipse depths in Figure 4 are too great to be mistaken for planetary transits.) To minimize resource-intensive spectroscopic follow-up work, we are planning to conduct our own high angular-resolution, multi-color photometry of transit candidates. To this end, we are currently building Sherlock (Kotredes et al. [2]), an automated follow-up telescope for wide-field transit searches.

\section{REFERENCES}

1. Kovács, G., Zucker, S., \& Mazeh, T. A\&A, 391, 369-377 (2002).

2. Kotredes, L., Charbonneau, D., O'Donovan, F. T., \& Looper, D. (2004), these proceedings. 